\title{
RNA Interference-Mediated Knockdown of Astrocyte Elevated Gene-1 Inhibits Growth, Induces Apoptosis, and Increases the Chemosensitivity to 5-Fluorouracil in Renal Cancer Caki-1 Cells
}

\author{
Peng Wang, Bo Yin, Liping Shan, Hui Zhang, Jun Cui, Mo Zhang, and Yongsheng Song*
}

\begin{abstract}
Astrocyte elevated gene-1 (AEG-1) is a recently discovered oncogene that has been reported to be highly expressed in various types of malignant tumors, including renal cell carcinoma. However, the precise role of AEG-1 in renal cancer cell proliferation and apoptosis has not been clarified. In this study, we transfected the renal cancer cell line Caki-1 with a plasmid expressing AEG-1 short hairpin RNA (shRNA) and obtained cell colonies with stable knockdown of AEG-1. We found that AEG-1 down-regulation inhibited cell proliferation and colony formation and arrested cell cycle progression at the sub-G1 and G0/G1 phase. Western blot analysis indicated that the expression of proliferating cell nuclear antigen (PCNA), cyclin D1 and cyclin E were significantly reduced following AEG-1 down-regulation. In addition, AEG-1 knockdown led to the appearance of apoptotic bodies in renal cancer cells, and the ratio of apoptotic cells significantly increased. Expression of the antiapoptotic factor $\mathrm{Bcl}-2$ was dramatically reduced, whereas the pro-apoptotic factors Bax, caspase-3 and poly (ADPribose) polymerase (PARP) were significantly activated. Finally, AEG-1 knockdown in Caki-1 cells remarkably suppressed cell proliferation and enhanced cell apoptosis in response to 5-fluorouracil (5-FU) treatment, suggesting that AEG-1 inhibition sensitizes Caki-1 cells to 5-FU. Taken together, our data suggest that AEG-1 plays an important role in renal cancer formation and development and may be a potential target for future gene therapy for renal cell carcinoma.
\end{abstract}

\section{INTRODUCTION}

Kidney cancer or renal cell carcinoma (RCC) is a type of malig-

The Second Department of Urology, Shengjing Hospital of China Medical University, Shenyang 110004, People's Republic of China

*Correspondence: songyongsheng@outlook.com

Received 7 April, 2014; revised 18 August, 2014; accepted 18 September, 2014; published online 26 November, 2014

Keywords: apoptosis, astrocyte elevated gene-1, chemosensitivity, proliferation, renal cancer cells nant tumor originating from the epithelial cells of the renal tubule or collecting duct in the kidney. RCC is the most common malignant phenotype in the adult urinary system, with an incidence rate second only to bladder cancer (Gupta et al., 2008; Leibovich et al., 2010). Because RCC is resistant to traditional chemo- and radiotherapy treatments, surgical removal remains the primary treatment option. However, RCC has a poor prognosis, and approximately $25 \%$ of RCC patients experience recurrence and metastasis after surgery (Dutcher, 2013). Therefore, a thorough understanding of RCC oncogenesis may aid in the identification of a more effective target for RCC gene therapy and provide an important theoretical and practical basis for developing more effective therapeutic means that can prolong patient survival.

Astrocyte elevated gene-1 (AEG-1) was discovered as an HIV-1- or tumor necrosis factor- $\alpha$-inducible gene in primary human fetal astrocytes by Su et al. in 2002 (Su et al., 2003). AEG-1 is primarily located in the perinuclear region and endoplasmic reticulum (Sutherland et al., 2004) and was initially discovered to be significantly upregulated in glioblastoma (Kang et al., 2005). Recent studies confirmed that AEG-1 shows an abnormally high expression in many malignant tumors (Liu et al., 2010; Yoo et al., 2009a; Yu et al., 2009) and participates in the major pathways of tumorigenesis, including cellular transformation, apoptosis inhibition, invasion, metastasis, angiogenesis and resistance to chemotherapeutic agents (Emdad et al., 2009; 2010; Gnosa et al., 2012; Jiang et al., 2012; Ke et al., 2013; Li et al., 2011; Song et al., 2010; Sun et al., 2011; Xia et al., 2010; Yoo et al., 2010). In addition, a recent study by Chen et al. (2010) indicates that AEG-1 is highly expressed in the majority of RCC tissues and relates to tumor grading and progression. Nevertheless, the exact role of AEG-1 in RCC formation and development remains unclear.

In the present study, we constructed a plasmid-based RNA interference specifically targeting AEG-1, which was then used for transfecting the renal cancer cell line Caki- 1 in vitro. In stably transfected cell colonies, we examined the effect of AEG-1 on RCC cell proliferation, colony formation, cell cycle and apoptosis. The results showed that stable knockdown of AEG-1 inhibited RCC cell proliferation and colony formation, arrested the cell cycle at the G0/G1 phase, eventually induced cell apoptosis, and also increased the chemosensitivity. Thus, AEG-1 plays an important role in RCC formation and development and might be a potential target for RCC gene therapy. 


\section{MATERIALS AND METHODS}

\section{Cell culture}

The human renal cancer Caki-1 cells were obtained from the Cell Bank of Chinese Academy of Sciences (China) and cultured in McCOY's 5A media (Sigma-Aldrich, USA) containing $10 \%$ fetal bovine serum (FBS; Hyclone, USA) at $37^{\circ} \mathrm{C}$ in a $5 \%$ $\mathrm{CO}_{2}$ atmosphere Culture media were replaced every 2-3 days until the cells reached $80 \%$ confluence, and $0.25 \%$ trypsin was then added for cell passaging. 5-fluorouracil (5-FU, purity > 98\%) was purchased from Meilun Biotechnology (China).

\section{Plasmid construction}

The pGCsi-H1 vector (GeneChem, China) was utilized for constructing the specific AEG-1 shRNA-expressing plasmid. After locating the AEG-1 mRNA sequence in the NCBI database (NM_178812.3), the target sequence GGTGAAGATAACTCTACT $G$ was selected based on the shRNA design guideline. The primers used for shRNA synthesis are as follows: forward 5'-GATCCCCGGTGAAGATAACTCTACTGTTCAAGAGACAGTAGAGTTATCTTCACCTTTTT-3' and reverse 5'-AGCTAAAAAGGTGAAGATAACTCTACTGTCTCTTGAACAGTAGAGTT ATCTTCACCGGG-3'. Following annealing, the above two strands of oligonucleotides were subcloned into the pGCsi-H1 vector via HindIII and BamHI restriction sites. Meanwhile, a sequence sharing a similar size but no homology with human genome sequences was inserted into $\mathrm{pGCsi-H1}$ vector to serve as a negative control. The AEG-1 shRNA-expressing plasmid was confirmed via double enzyme digestion and sequencing prior to proceeding to the subsequent experiments.

Cell transfection and screening of stably transfected cell clones

Caki-1 cells were seeded at a density of $1.0 \times 10^{5}$ per well in 6well plates and incubated overnight. Upon reaching $70-80 \%$ confluence, the cells were transfected with pGCsi-H1 containing AEG-1-targeted shRNA or negative control using Lipofectamine 2000 (Invitrogen, USA). The cells were then treated with 800 $\mu \mathrm{g} / \mathrm{mL}$ geneticin (G418; Invitrogen) to screen for the stably transfected cell colonies through a continuous culturing of 4 weeks. Single colonies with G418 resistance were selected and confirmed for low AEG-1 expression. Confirmed colonies were then continually cultured in media containing $350 \mu \mathrm{g} / \mathrm{ml}$ G418 and expanded for the ensuing studies.

\section{Real-time PCR}

Total RNA from individual groups was extracted using the TRIzol reagent (Invitrogen) and reverse transcribed into cDNA using First Strand cDNA Synthesis Kit (Takara, China). All steps were performed rigorously according to the kit protocol. The forward and reverse primers for amplifying AEG-1 are as follows: 5'-ATCCTACCTATCTGTACCGAC-3' and 5'-GGGTGAATTATTCTGACCTCC-3'. The length of the amplified fragment was $144 \mathrm{bp}$. A 156-bp fragment of $\beta$-actin was used as the internal control and was amplified with the following forward and reverse primers: $5^{\prime}$-CTTAGTTGCGTTACACCCTTTCTTG$3^{\prime}$ and 5'-CTGTCACCTTCACCGTTCCAGTTT-3'. Real-time PCR was performed on an Exicycler ${ }^{\top M} 96$ fluorescent quantitative thermo block (Bioneer, Korea) in a $20-\mu$ reaction comprising $1 \mu \mathrm{l}$ of cDNA, $0.5 \mu \mathrm{l}$ of forward primer, $0.5 \mu \mathrm{l}$ of reverse primer, $10 \mu$ of SYBR GREEN mastermix and $8 \mu$ of $\mathrm{ddH}_{2} \mathrm{O}$. The PCR cycling conditions are as follows: $95^{\circ} \mathrm{C}$ for $10 \mathrm{~min} ; 40$ cycles of $95^{\circ} \mathrm{C}$ for $10 \mathrm{~s}, 58^{\circ} \mathrm{C}$ for $20 \mathrm{~s}$ and $72^{\circ} \mathrm{C}$ for $30 \mathrm{~s}$; and $4^{\circ} \mathrm{C}$ for $5 \mathrm{~min}$.

\section{Western blotting}

Cells were lysed using RIPA lysis buffer (Beyotime Institute of Biotechnology, China). The protein concentrations were determined using the bicinchoninic acid assay (BCA). A total of $40 \mu \mathrm{g}$ of proteins was electrophoresed via sodium dodecyl sulphate polyacrylamide gel electrophoresis (SDS-PAGE), and transferred onto polyvinylidene fluoride (PVDF) membranes (Millipore, USA), which were then blocked with $5 \%$ non-fat dry milk for $1 \mathrm{~h}$ at room temperature. The membranes were incubated with antibodies against AEG-1 (1:1000 dilution; Abcam, USA), Bcl-2 (1: 100 dilution), $\operatorname{Bax}$ (1: 100 dilution), caspase-3 (1: 100 dilution; Biosynthesis Biotechnology, China), PCNA (1: 500 dilution), Cyclin D1 (1: 200 dilution), Cyclin E (1: 200 dilution), PARP (1: 500 dilution; Santa Cruz Biotechnology, USA), followed by incubation with corresponding horseradish peroxidase (HRP)-conjugated secondary antibody (Beyotime Institute of Biotechnology) at a 1:5000 dilution for $1 \mathrm{~h}$ at $37^{\circ} \mathrm{C}$. Chemiluminescence was then produced by chemiluminescence substrate (Thermo Scientific Pierce) and subsequently exposed to X-film and scanned into the computer. Protein expression levels from each group were determined via gray scale analysis using Image $\mathrm{J}$ software. The $\beta$-actin served as an internal control.

\section{Immunofluorescence}

Caki-1 cells were placed on a cover slip in a petri dish and grown to confluency. The cells were then fixed with $4 \%$ paraformaldehyde and incubated overnight at $4^{\circ} \mathrm{C}$. After fixing, the coverslip was incubated with $1 \%$ Triton $\mathrm{X}-100$ for $8 \mathrm{~min}$, and then replaced with $3 \% \mathrm{H}_{2} \mathrm{O}_{2}$. Blocking was performed using goat serum, following by incubation with primary AEG-1 antibody (Abcam) at a 1:200 dilution overnight at $4^{\circ} \mathrm{C}$. PE-labeled fluorescent secondary antibody (Abcam) was then incubated with the coverslip at $37^{\circ} \mathrm{C}$ for $30 \mathrm{~min}$. Cell nuclei were stained with 4',6-diamidino-2-phenylindole (DAPI). The coverslips were then mounted by adding anti-fade fluorescence medium and subjected to laser scanning confocal microscopy (FV1000SSIM/IX81; Olympus, Japan) for further observation and imaging.

\section{Cell proliferation assay}

Cell proliferation was evaluated by the 3-(4,5-dimethylthiazol-2yl)-2,5-diphenyltetrazolium bromide (MTT) colorimetric assay. In brief, cells were counted on a hemocytometer and seeded in a 96-well plate at a density of $5 \times 10^{3}$ per well. When cells become confluent, $100 \mu \mathrm{l}$ of MTT $(0.5 \mathrm{mg} / \mathrm{ml}$; Sigma-Aldrich, USA) was added at different time points for a continual incubation of $4 \mathrm{~h}$ at $37^{\circ} \mathrm{C}$. After removing the supernatants, $200 \mu \mathrm{l}$ of dimethyl sulfoxide (DMSO; Sigma-Aldrich) was added to dissolve the formazan crystals in the cells. Absorbance at $490 \mathrm{~nm}$ by cells was then measured using a spectrophotometer.

\section{Colony formation assay}

The cells were seeded in 10-mm plates at a density of $2 \times$ $10^{2} /$ plate and cultured at $37^{\circ} \mathrm{C}$ for $10-14$ days until visible cell colonies appeared. The cells were then washed with phosphate buffered saline (PBS) twice and fixed with methanol. After removing the methanol, the cell colonies were stained with Giemsa solution for $10 \mathrm{~min}$; then, the Giemsa was removed, and the colonies were counted.

Cell cycle and apoptosis analysis by flow cytometry For cell cycle analysis, cells were trypsinized and fixed in precooled $70 \%$ ethanol overnight. Propidium iodide (PI) containing RNase $(50 \mu \mathrm{g} / \mathrm{ml})$ was then added to the cells and incubated at $37^{\circ} \mathrm{C}$ for $30 \mathrm{~min}$. The percentages of cells at different phases 
A
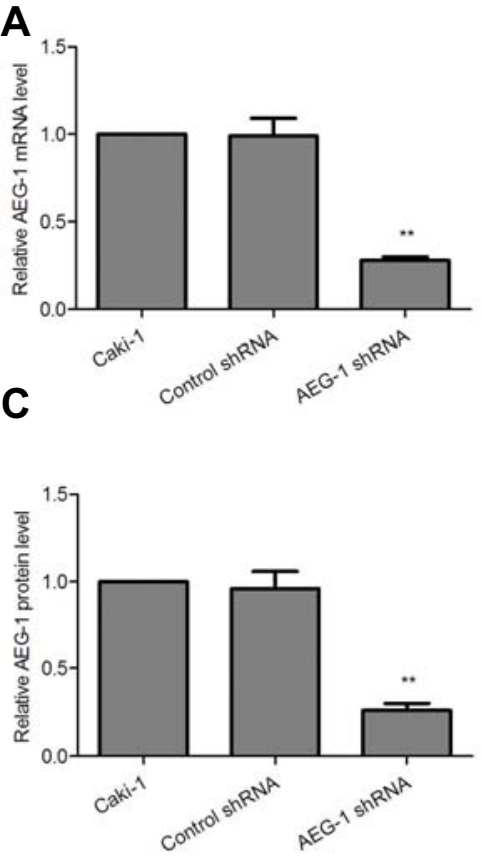

B

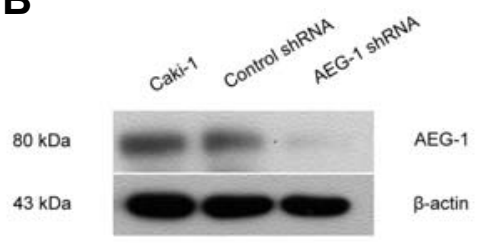

D

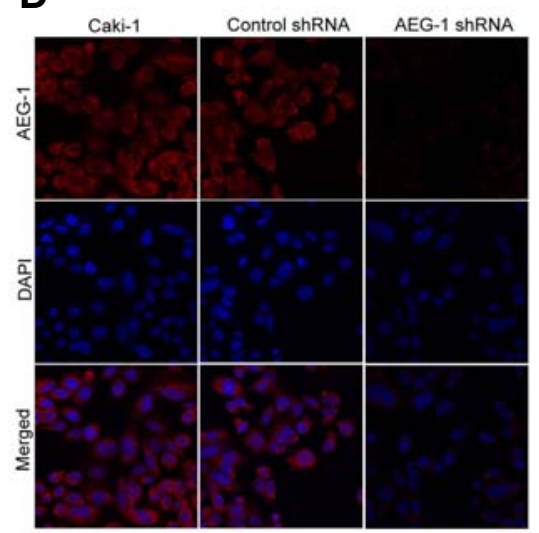

Fig. 1. RNAi-mediated AEG-1 down-regulation in renal cancer cells. AEG-1 mRNA and protein expression levels were examined by realtime PCR (A) and Western blot analysis (B), respectively. The $\beta$-actin was used as an internal control for the densitometric analysis (C). AEG-1 expression was analyzed by immunofluorescence (D). AEG-1 (red) was primarily expressed within the cytoplasm and in regions surrounding the outside of nuclei (blue). The images shown are representative results. ${ }^{* *} \mathrm{P}<0.01$ compared with the control shRNA cells. were then assessed through flow cytometry (BD Biosciences, USA).

The cell apoptosis rates were analyzed using an Annexin VFITC/PI apoptosis kit (KeyGen Biotech, China). When reaching $80 \%$ confluency, the cells were trypsinized, washed with PBS and resuspended in $500 \mu \mathrm{l}$ of Annexin V-binding buffer. A mixture of $5 \mu \mathrm{l}$ of FITC-labeled Annexin V plus $5 \mu \mathrm{l}$ of PI solution was then added into the cells to incubate for $15 \mathrm{~min}$ at room temperature in the dark prior to flow cytometry analysis.

\section{Hoechst 33258 staining}

Hoechst 33258 staining was performed to detect the changes in cell nuclei and formation of apoptotic bodies. Upon reaching $80 \%$ confluency, the cells were fixed by $4 \%$ paraformaldehyde for 20 min, washed with PBS, and incubated with Hoechst 33258 solution (Beyotime Institute of Biotechnology) at room temperature for $30 \mathrm{~min}$. The staining solution was then discarded, and the cover slips were mounted with anti-fade fluorescence medium for further observation and imaging using laser scanning confocal microscopy.

\section{Statistical analysis}

All experiments were repeated three times, and the data are expressed as the means \pm standard deviation. Differences among groups were analyzed using one-way analysis of variance, and Bonferroni post-hoc analysis was performed for multiple comparisons. Graphpad Prism 5.0 was utilized to plot data and generate graphs. $P<0.05$ indicates the difference was significant.

\section{RESULTS}

Generation of the Caki-1 cells with stable knockdown of AEG-1

To investigate the role of AEG-1 in renal cancer, we constructed plasmids expressing AEG-1 shRNA or negative control shRNA.
After transfecting Caki-1 cells with these plasmids, we screened and obtained G418-resistant cell colonies and examined their AEG-1 expression by real-time PCR and Western blot analysis. The results showed that AEG-1 shRNA mediated AEG-1 mRNA and protein expression knockdown by 72 and $74 \%$, respectively (Figs. $1 \mathrm{~A}-1 \mathrm{C}$; $\mathrm{P}<0.01$ ). Immunofluorescence analysis indicated that AEG-1 was primarily expressed in the cytoplasmic and perinuclear region. Compared with the control cells, AEG-1 shRNA-transfected cells exhibited a marked reduction in AEG-1 fluorescence intensity (Fig. 1D). The above results suggest that the plasmid expressing AEG-1-specific RNA interference effectively silenced AEG-1 expression at both the transcriptional and translational levels and thus served as a valid tool for our subsequent studies.

AEG-1 knockdown inhibits the proliferation and colony formation of Caki-1 cells

We investigated the effects of AEG-1 knockdown on Caki- 1 cell proliferation using the MTT assay. As shown in Fig. 2A, the proliferative ability of AEG-1 shRNA-transfected cells was markedly reduced $72 \mathrm{~h}$ after transfection and was significantly lower than the control group following 96 or $120 \mathrm{~h}$ of incubation $(\mathrm{P}<0.05)$. Next, we assessed the effects of AEG-1 knockdown on anchorage-independent growth by the colony formation assay. Compared with the control cells, the number of colonies formed by AEG-1 shRNA-transfected cells was significantly decreased (Figs. 2B and 2C; $\mathrm{P}<0.01$ ). Furthermore, Western blot analysis showed that PCNA expression was significantly reduced following AEG-1 knockdown in Caki-1 cells (Figs. 2D and 2E; P < 0.01). Taken together, our data suggest that AEG-1 is involved in cell proliferation and in vitro tumorigenic potential of Caki-1 cells.

AEG-1 knockdown induces cell cycle arrest at the G0/G1 phase in Caki- 1 cells

To better understand the mechanisms underlying the regulation 


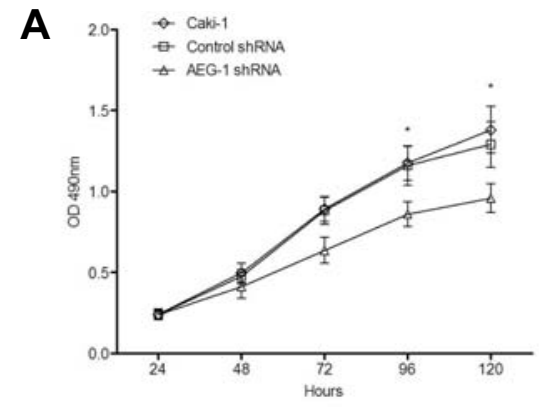

B

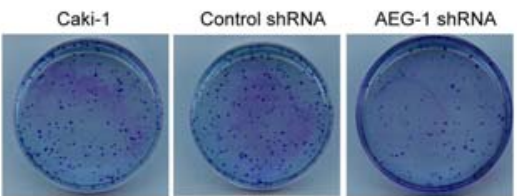

D

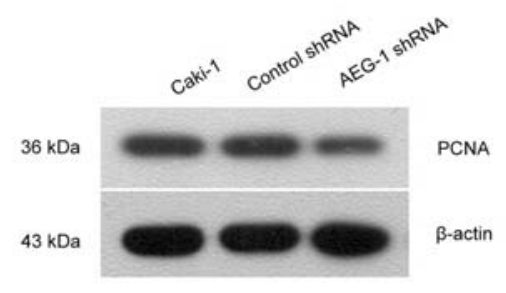

C

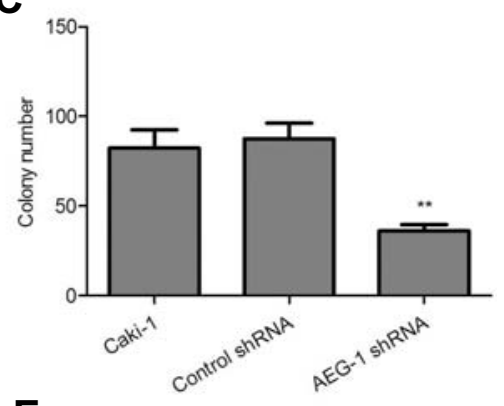

E

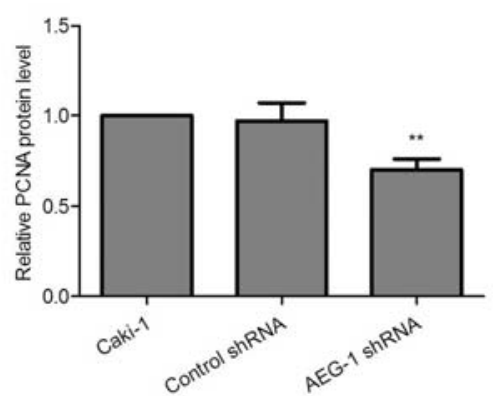

Fig. 2. AEG-1 knockdown inhibits cell proliferation and colony formation in Caki-1 cells. (A) The MTT assay was performed to examine cell proliferation. The cells were seeded into 96-well plates, and the absorbance at $490 \mathrm{~nm}$ was measured at the indicated time points; (B) The anchorageindependent growth was assessed by the colony formation assay. The cells were seeded in $10-\mathrm{mm}$ plates at a density of $2 \times$ $10^{2} /$ well. (C) The number of colonies was counted after 10-14 days. (D) PCNA protein expression was analyzed using Western blotting. Representative results are shown. (E) Densitometric values were normalized by $\beta$-actin. ${ }^{*} P<0.05$, ${ }^{* *} P<0.01$ compared with the control shRNA cells. of cell proliferation by AEG-1, we examined the effects of AEG1 knockdown on the cell cycle. As illustrated in Figs. 3A and 3B, there was a marked increase in the number of cells at the G0/G1 phase in cells receiving AEG-1 shRNA transfection compared with control cells $(P<0.01)$. Meanwhile, the percentage of cells in $S$ phase was significantly decreased $(P<0.01)$. Furthermore, the proportion of sub-G1 apoptotic cells was also dramatically increased after AEG-1 shRNA transfection ( $P$ < 0.01). Western blot analysis indicated a significant reduction in the expression of Cyclin D1 and Cyclin E in AEG-1 shRNAtransfected cells in comparison to control cells (Figs. 3C and 3D; $P<0.01$ ). Therefore, AEG-1 down-regulation arrests cells at the G0/G1 phase, thereby inhibiting cell proliferation.

AEG-1 knockdown promotes apoptosis in Caki-1 cells We then used Hoechst staining and flow cytometry to determine the effects of AEG-1 knockdown on cell apoptosis. As shown in representative results from Hoechst staining Fig. 4A, the cells transfected with AEG-1 shRNA contained apparent apoptotic bodies, whereas few were observed in the control cells. Figures $4 \mathrm{~B}$ and $4 \mathrm{C}$ show the percentages of apoptotic cells as measured by Annexin V-FITC/PI, in which the percentage of apoptotic cell population in AEG-1 shRNA-transfected cells (19.91 $\pm 4.85 \%)$ was clearly higher than in those transfected with control shRNA $(5.29 \pm 1.47 \%)$ and in the non-transfected control $(4.58 \pm 1.36 \%)$. Moreover, Western blot analysis showed that AEG-1 shRNA significantly decreased $\mathrm{Bcl}-2$ expression levels and increased the expression of Bax, cleaved PARP and caspase- 3 in Caki-1 cells (Figs. 4D and 4E; $P<0.01$ ). Hence, our observations suggest that suppression of cell growth by AEG-1 shRNA is partially attributable to increased apoptosis in vitro.

AEG-1 knockdown enhances the chemosensitivity to 5-FU in Caki-1 cells

To further investigate whether AEG-1 knockdown in Caki- 1 cells affects their sensitivity to 5-FU, control cells and AEG-1 shRNAtransfected cells were treated with increasing concentrations of 5 -FU for $48 \mathrm{~h}$ and then analyzed for viability by the MTT assay. We found that 5-FU treatment caused concentration-dependent growth inhibition, and the inhibition rates were obviously enhanced in AEG-1 shRNA-transfected cells compared with those in control cells (Fig. 5A). More importantly, combined AEG-1 shRNA and 5-FU treatment led to a significant decrease in IC50 value $(22 \mu \mathrm{g} / \mathrm{ml})$ in comparison to 5 -FU treatment $(43 \mu \mathrm{g} / \mathrm{ml}$ in Caki- 1 cells and $40 \mu \mathrm{g} / \mathrm{ml}$ in control shRNA-transfected cells). Next, the cells were treated with $22 \mu \mathrm{g} / \mathrm{ml} 5-\mathrm{FU}$ and harvested for cell proliferation and apoptosis. Compared with control cells, AEG-1 shRNA cells displayed increased sensitivity to 5-FU, as indicated by the MTT assay (Fig. 5C) and flow cytometry analysis (Fig. 5D). Altogether, these findings strongly suggest that AEG-1 is involved in modulating 5-FU cytotoxicity in Caki-1 cells. 
A

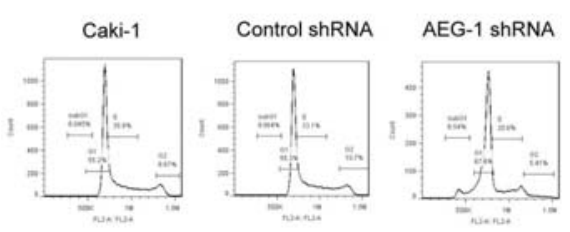

C

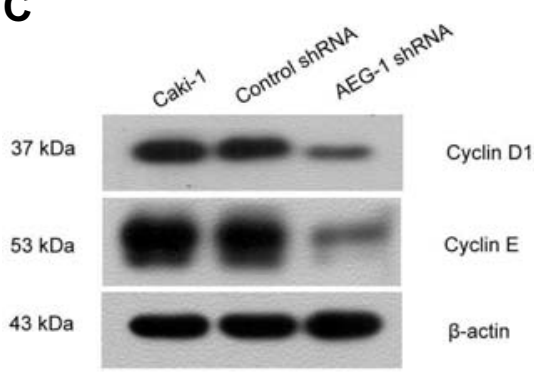

B

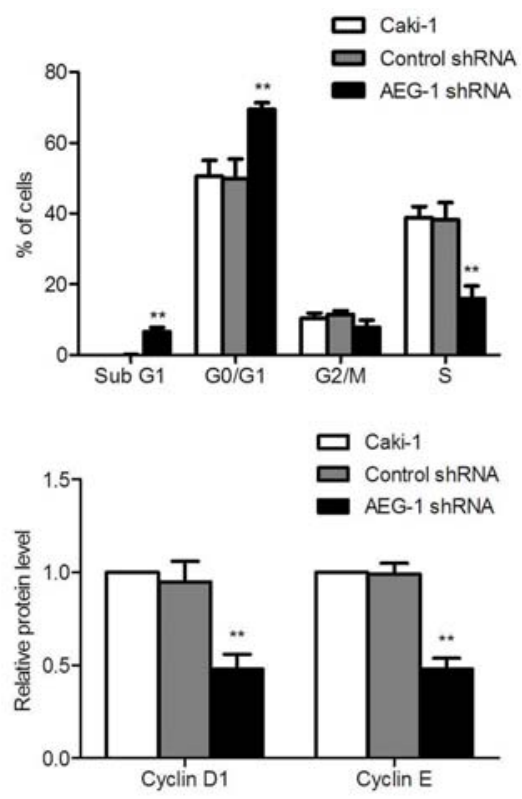

A
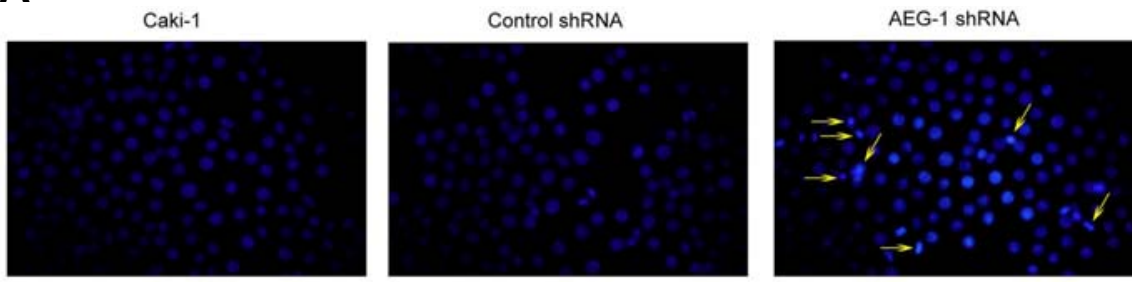

B

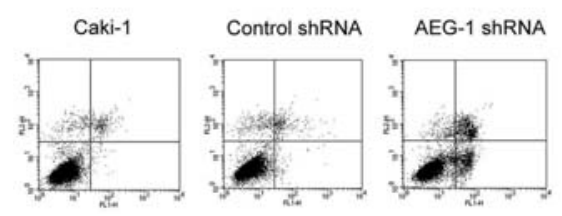

D

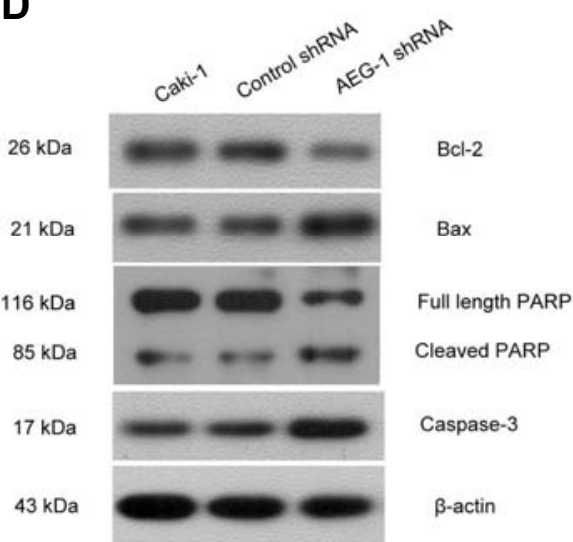

C

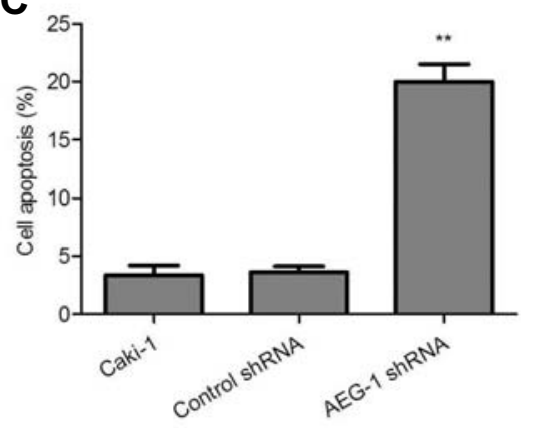

E

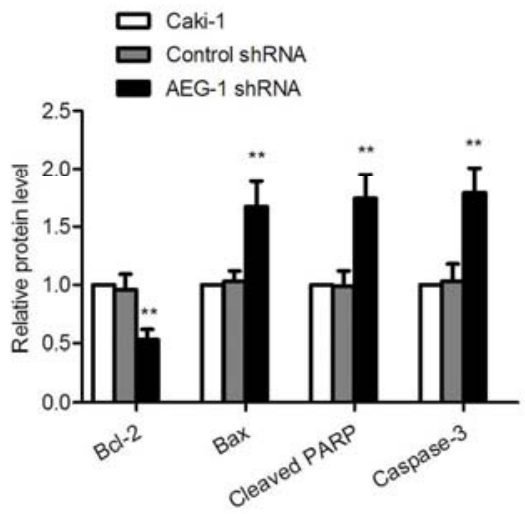

Fig. 3. AEG-1 knockdown arrests the cell cycle at G0/G1 in Caki-1 cells. (A) Cell cycle was examined by flow cytometry. Representative results are shown. PI staining was performed when the cells reached $80 \%$ confluency. (B) The percentages of cells at each phase were quantified. (C) The expression levels of Cyclin D1 and Cyclin E were detected by Western blot analysis. Representative blots are shown. (D) Quantitative data are expressed as the intensity ratio of Cyclin D1 or Cyclin $E$ to $\beta$-actin. ${ }^{* *} P<0.01$ compared with the control shRNA cells.

Fig. 4. AEG-1 knockdown induces cell apoptosis in Caki-1 cells. (A) Cell apoptosis was assessed using Hoechst 33258 staining and confocal imaging. Representative images are shown. (B) Cell apoptosis was examined by Annexin V-PI staining. (C) The number of $\mathrm{PI} /$ Annexin $\mathrm{V}$ single positive and Annexin V/PI double positive cells was calculated as the measurement of apoptotic cells. (D) The protein expression levels of Bcl-2, Bax, PARP and caspase-3 were examined by Western blot analysis. Representative blots are shown and protein size is expressed in $\mathrm{kDa}$. The $\beta$-actin was used as a loading control. (E) Quantitative analysis was performed by densitometry. ${ }^{*} \mathrm{P}<0.01$ compared with the control shRNA cells. 


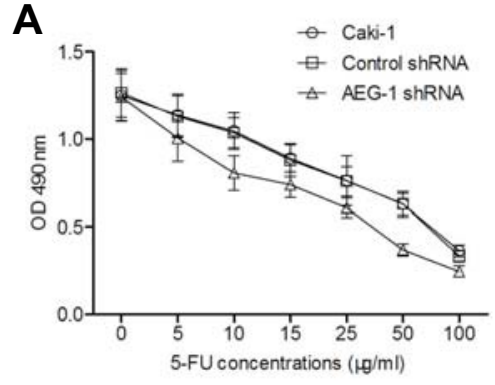

C

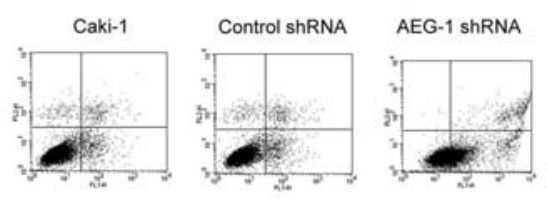

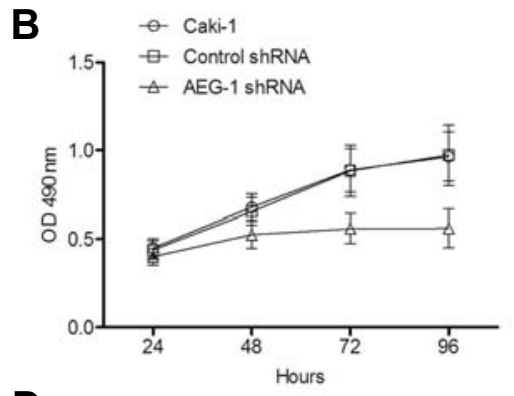

D

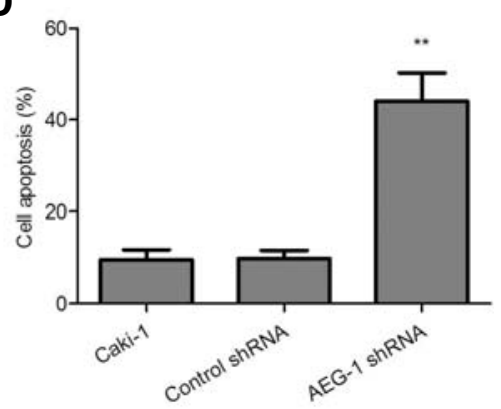

Fig. 5. AEG-1 knockdown increases the chemosensitivity to 5 -fluorouracil in Caki-1 cells. (A) Cells were treated with different concentrations of 5-FU for $48 \mathrm{~h}$, and cell viability was assessed by the MTT assay. (B, C) Cells were treated with $22 \mu \mathrm{g} / \mathrm{ml} 5$ FU. Cell viability and apoptosis were measured by the MTT assay (B) and the flow cytometry analysis (C), respectively. (D) The percentage of apoptotic cells was quantified. ${ }^{* *} \mathrm{P}<0.01$ compared with the control shRNA cells.

\section{DISCUSSION}

Clinical studies have demonstrated that there is an intimate relationship between abnormally high AEG-1 expression levels and a poor prognosis for kidney cancer patients (Erdem et al., 2013). However, the exact role of AEG-1 in RCC proliferation and apoptosis has not been clarified. In this study, we constructed a plasmid-based RNA interference molecule specifically targeting AEG-1, transfected it into the renal cancer cell line Caki-1 and subsequently obtained stably transfected cells via G418 selection. The ability of AEG-1 shRNA-expressing plasmid to specifically reduce AEG-1 expression was confirmed by real-time PCR, Western blot and immunofluorescence analysis. Further studies revealed that AEG-1 knockdown inhibited Caki1 cell proliferation and colony formation, arrested the cells at the G0/G1 phase, reduced Cyclin D1 and Cyclin E expression, and eventually led to apoptosis. Consistent with the above results, the expression levels of Bax, cleaved PARP and caspase- 3 were significantly increased following AEG-1 knockdown. Our results suggest that AEG-1 plays an important role in RCC formation and development.

The AEG-1 gene was initially discovered in primary human astrocytes and has been suggested to promote neurodegeneration (Su et al., 2003). Recent studies reveal that AEG-1 promotes tumor progression and serves as a key oncogene during tumor formation and development. Aberrantly high AEG-1 expression levels closely correlate with the poor prognosis of cervical cancer (Huang et al., 2013a), gastric cancer (Huang et al., 2013b), and neuroblastoma (Liu et al., 2012), whereas AEG-1 downregulation inhibits cancer cell proliferation and tumorigenicity (Deng et al., 2014; Li et al., 2009; Liu et al., 2009). In the present study, we used RNA interference to knockdown AEG-1 expression in renal cancer cells and found that AEG-1 knockdown resulted in the apparent inhibition of cell proliferation and colony formation. Furthermore, PCNA expression was substantially decreased following AEG-1 down-regulation. Our data suggest that AEG-1 has an important role in RCC, and inhibiting AEG-1 can lower tumor malignancy.

Cyclin D1 is a key protein that regulates the transition of cells from $\mathrm{G} 1$ to $S$ phase during cell cycle progression (Duman-Scheel et al., 2002); Cyclin D1 activation promotes the passage of cells through the G1 restriction checkpoint (Moroy and Geisen, 2004). In previous studies, RNAi-mediated AEG-1 silencing significantly increased the percentage of renal cancer cells arrested at the G0/G1 phase, which correlates with a substantial reduction in Cyclin D1 expression (Zhang et al., 2013a). Similarly, we observed a significant increase in the percentage of G0/G1 cells and a concomitant decrease in the percentage of cells in $S$ phase. In addition, there was a significant downregulation in the expression of Cyclin D1 and Cyclin E. These results suggest that downregulation of AEG-1 halted cell cycle progression at G0/G1 by inhibiting the expression of Cyclin D1 and Cyclin E. Similar conclusions were also reached from previous studies of colorectal cancer cells (Huang et al., 2014) and neuroblastomas (Liu et al., 2009). However, reducing AEG-1 did not alter the cell cycle distribution of the bladder cancer cells RT112 and 647V (Nikpour et al., 2014), suggesting that the influence of AEG-1 on the cell cycle may be cell- or tissue type-dependent.

Cell apoptosis is necessary during normal tissue development. Escaping apoptosis is one of the major hallmarks of cancer cells during tumorigenesis. Zhang et al. (2013b) have reported that AEG-1 not only inhibits the intrinsic apoptotic pathway by up-regulating Bcl-2 expression but also inhibits the extrinsic apoptotic pathway through regulating the caspase-8/3PARP pathway in breast cancer cells. In our study, decreasing AEG-1 promoted the formation of apoptotic cell bodies and increased the spontaneous apoptosis of Caki-1 cells. Meanwhile, AEG-1 down-regulation decreased the anti-apoptotic factor Bcl-2 while increasing the expression of the pro-apoptotic Bax, cleaved PARP and caspase-3. Additionally, Sub-G1 phase cells are considered to be resulted from apoptotic DNA fragmentation and are therefore usually used to evaluate the relative amount of apoptotic cells (Elstein and Zucker, 1994; Riccardi and Nicoletti, 2006). As expected, our data showed that the proportion of sub-G1 apoptotic cells was increased in cells transfected with AEG-1 shRNA. These results suggest that AEG-1 knockdown induces cell apoptosis through multiple pathways. However, the exact mechanism underlying the 
above observation requires further investigation.

Several lines of evidence indicate that increased expression of AEG-1 is associated with postoperative radioresistance or chemoresistance in patients with ovarian and rectal cancers (Gnosa et al., 2014; Li et al., 2012). However, whether AEG-1 contributes to chemoresistance in renal cancer remains to be elucidated. In the present study, we demonstrated that AEG-1 knockdown in Caki-1 cells remarkably suppressed cell proliferation and enhanced cell apoptosis in response to 5-FU treatment, suggesting that AEG-1 inhibition sensitizes Caki-1 cells to 5-FU. Our data are consistent with previous studies showing that AEG-1 increases the expression of multidrug resistance gene 1 (MDR1) and up-regulates the expression of the transcription factor LSF and thymidylate synthase (TS), a target of 5-FU, thereby contributing to multidrug resistance in hepatocellular carcinoma cells (Yoo et al., 2009b; 2010). Collectively, these findings imply that AEG-1 inhibition by RNAi along with 5-FUbased chemotherapy may be exploited as a potential synergistic therapy for patients with renal cancer.

Taken together, the present study demonstrated that AEG-1 knockdown inhibited cell growth, arrested cell cycle at the G0/G1 phase, promoted spontaneous apoptosis, and enhanced the chemosensitivity to 5-fluorouracil in renal cancer Caki-1 cells. Our data suggest that AEG-1 may serve as a promising therapeutic target for renal cancer.

\section{ACKNOWLEDGMENTS}

This study was supported by a grant from the Natural Science Foundation of Liaoning Province (No.: 201102286).

\section{REFERENCES}

Chen, W., Ke, Z., Shi, H., Yang, S., and Wang, L. (2010). Overexpression of AEG-1 in renal cell carcinoma and its correlation with tumor nuclear grade and progression. Neoplasma 57, 522-529.

Deng, H., Zhou, Z., Tu, W., Xia, Y., Huang, H., and Tian, D. (2014). Knockdown of astrocyte elevated gene-1 inhibits growth through suppression of IL-6 secretion in HepG2 human hepatoma cells. Oncol. Lett. 7, 101-106.

Duman-Scheel, M., Weng, L., Xin, S., and Du, W. (2002). Hedgehog regulates cell growth and proliferation by inducing cyclin D and cyclin E. Nature 417, 299-304.

Dutcher, J.P. (2013). Recent developments in the treatment of renal cell carcinoma. Ther Adv. Urol. 5 , 338-353.

Elstein, K.H., and Zucker, R.M. (1994). Comparison of cellular and nuclear flow cytometric techniques for discriminating apoptotic subpopulations. Exp. Cell Res. 211, 322-331.

Emdad, L., Lee, S.G., Su, Z.Z., Jeon, H.Y., Boukerche, H., Sarkar, D., and Fisher, P.B. (2009). Astrocyte elevated gene-1 (AEG-1) functions as an oncogene and regulates angiogenesis. Proc. Natl. Acad. Sci. USA 106, 21300-21305

Emdad, L., Sarkar, D., Lee, S.G., Su, Z.Z., Yoo, B.K., Dash, R., Yacoub, A., Fuller, C.E., Shah, K., Dent, P., et al. (2010). Astrocyte elevated gene-1: a novel target for human glioma therapy. Mol. Cancer Ther. 9, 79-88.

Erdem, H., Oktay, M., Yildirim, U., Uzunlar, A.K., and Kayikci, M.A. (2013). Expression of AEG-1 and p53 and their clinicopathological significance in malignant lesions of renal cell carcinomas: a microarray study. Pol. J. Pathol. 64, 28-32.

Gnosa, S., Shen, Y.M., Wang, C.J., Zhang, H., Stratmann, J. Arbman, G., and Sun, X.F. (2012). Expression of AEG-1 mRNA and protein in colorectal cancer patients and colon cancer cell lines. J. Transl. Med. 10, 109.

Gnosa, S., Zhang, H., Brodin, V.P., Carstensen, J., Adell, G., and Sun, X.F. (2014). AEG-1 expression is an independent prognostic factor in rectal cancer patients with preoperative radiotherapy: a study in a Swedish clinical trial. Br. J. Cancer 111, 166-173.

Gupta, K., Miller, J.D., Li, J.Z., Russell, M.W., and Charbonneau, C. (2008). Epidemiologic and socioeconomic burden of metastatic renal cell carcinoma $(\mathrm{mRCC})$ : a literature review. Cancer Treat. Rev. 34, 193-205.

Huang, K., Li, L.A., Meng, Y., You, Y., Fu, X., and Song, L. (2013a). High expression of astrocyte elevated gene-1 (AEG-1) is associated with progression of cervical intraepithelial neoplasia and unfavorable prognosis in cervical cancer. World J. Surg. Oncol. 11, 297.

Huang, W., Yang, L., Liang, S., Liu, D., Chen, X., Ma, Z., Zhai, S., Li, P., and Wang, X. (2013b). AEG-1 is a target of perifosine and is over-expressed in gastric dysplasia and cancers. Dig. Dis. Sci. $58,2873-2880$.

Huang, S., Wu, B., Li, D., Zhou, W., Deng, G., Zhang, K., and Li, Y. (2014). Knockdown of astrocyte elevated gene-1 inhibits tumor growth and modifies microRNAs expression profiles in human colorectal cancer cells. Biochem. Biophys. Res. Commun. 444, 338-345.

Jiang, T., Zhu, A., Zhu, Y., and Piao, D. (2012). Clinical implications of AEG-1 in liver metastasis of colorectal cancer. Med. Oncol. 29, 2858-2863.

Kang, D.C., Su, Z.Z., Sarkar, D., Emdad, L., Volsky, D.J., and Fisher, P.B. (2005). Cloning and characterization of HIV-1-inducible astrocyte elevated gene-1, AEG-1. Gene 353, 8-15

Ke, Z.F., Mao, X., Zeng, C., He, S., Li, S., and Wang, L.T. (2013). AEG-1 expression characteristics in human non-small cell lung cancer and its relationship with apoptosis. Med. Oncol. 30, 383.

Leibovich, B.C., Lohse, C.M., Crispen, P.L., Boorjian, S.A., Thompson, R.H., Blute, M.L., and Cheville, J.C. (2010). Histological subtype is an independent predictor of outcome for patients with renal cell carcinoma. J. Urol. 183, 1309-1315.

Li, J., Yang, L., Song, L., Xiong, H., Wang, L., Yan, X., Yuan, J., Wu, J., and Li, M. (2009). Astrocyte elevated gene-1 is a proliferation promoter in breast cancer via suppressing transcriptional factor FOXO1. Oncogene 28, 3188-3196.

Li, C., Liu, J., Lu, R., Yu, G., Wang, X., Zhao, Y., Song, H., Lin, P., Sun, X., Yu, X., et al. (2011). AEG -1 overexpression: a novel indicator for peritoneal dissemination and lymph node metastasis in epithelial ovarian cancers. Int. J. Gynecol. Cancer 21, 602-608.

Li, C., Li, Y., Wang, X., Wang, Z., Cai, J., Wang, L., Zhao, Y., Song, H., Meng, X., Ning, X., et al. (2012). Elevated expression of astrocyte elevated gene-1 (AEG-1) is correlated with cisplatinbased chemoresistance and shortened outcome in patients with stages III-IV serous ovarian carcinoma. Histopathology 60, 953963.

Liu, H., Song, X., Liu, C., Xie, L., Wei, L., and Sun, R. (2009). Knockdown of astrocyte elevated gene-1 inhibits proliferation and enhancing chemo-sensitivity to cisplatin or doxorubicin in neuroblastoma cells. J. Exp. Clin. Cancer Res. 28, 19.

Liu, L., Wu, J., Ying, Z., Chen, B., Han, A., Liang, Y., Song, L., Yuan, J., Li, J., and Li, M. (2010). Astrocyte elevated gene-1 upregulates matrix metalloproteinase- 9 and induces human glioma invasion. Cancer Res. 70, 3750-3759.

Liu, H.Y., Liu, C.X., Han, B., Zhang, X.Y., and Sun, R.P. (2012). AEG-1 is associated with clinical outcome in neuroblastoma patients. Cancer Biomark 11, 115-121.

Moroy, T., and Geisen, C. (2004). Cyclin E. Int. J. Biochem. Cell Biol. 36, 1424-1439.

Nikpour, M., Emadi-Baygi, M., Fischer, U., Niegisch, G., Schulz, W.A., and Nikpour, P. (2014). MTDH/AEG-1 contributes to central features of the neoplastic phenotype in bladder cancer. Urol. Oncol. 32, 670-677.

Riccardi, C., and Nicoletti, I. (2006). Analysis of apoptosis by propidium iodide staining and flow cytometry. Nat. Protoc. 1, 1458-1461.

Song, H., Li, C., Lu, R., Zhang, Y., and Geng, J. (2010). Expression of astrocyte elevated gene-1: a novel marker of the pathogenesis, progression, and poor prognosis for endometrial cancer. Int. J. Gynecol. Cancer 20, 1188-1196.

Su, Z.Z., Chen, Y., Kang, D.C., Chao, W., Simm, M., Volsky, D.J., and Fisher, P.B. (2003). Customized rapid subtraction hybridization ( $\mathrm{RaSH}$ ) gene microarrays identify overlapping expression changes in human fetal astrocytes resulting from human immunodeficiency virus-1 infection or tumor necrosis factor-alpha treatment. Gene 306, 67-78.

Sun, W., Fan, Y.Z., Xi, H., Lu, X.S., Ye, C., and Zhang, J.T. (2011). Astrocyte elevated gene-1 overexpression in human primary gallbladder carcinomas: an unfavorable and independent 
prognostic factor. Oncol. Rep. 26, 1133-1142.

Sutherland, H.G., Lam, Y.W., Briers, S., Lamond, A.I., and Bickmore, W.A. (2004). 3D3/lyric: a novel transmembrane protein of the endoplasmic reticulum and nuclear envelope, which is also present in the nucleolus. Exp. Cell Res. 294, 94-105.

Xia, Z., Zhang, N., Jin, H., Yu, Z., Xu, G., and Huang, Z. (2010). Clinical significance of astrocyte elevated gene-1 expression in human oligodendrogliomas. Clin. Neurol. Neurosurg. 112, 413419.

Yoo, B.K., Emdad, L., Su, Z.Z., Villanueva, A., Chiang, D.Y. Mukhopadhyay, N.D., Mills, A.S., Waxman, S., Fisher, R.A Llovet, J.M., et al. (2009a). Astrocyte elevated gene-1 regulates hepatocellular carcinoma development and progression. J. Clin. Invest. 119, 465-477.

Yoo, B.K., Gredler, R., Vozhilla, N., Su, Z.Z., Chen, D., Forcier, T., Shah, K., Saxena, U., Hansen, U., Fisher, P.B., et al. (2009b) Identification of genes conferring resistance to 5-fluorouracil. Proc. Natl. Acad. Sci. USA 106, 12938-12943.

Yoo, B.K., Chen, D., Su, Z.Z., Gredler, R., Yoo, J., Shah, K., Fisher, P.B., and Sarkar, D. (2010). Molecular mechanism of chemoresistance by astrocyte elevated gene-1. Cancer Res. 70, 3249-3258.

Yu, C., Chen, K., Zheng, H., Guo, X., Jia, W., Li, M., Zeng, M., Li, J., and Song, L. (2009). Overexpression of astrocyte elevated gene-1 (AEG-1) is associated with esophageal squamous cell carcinoma (ESCC) progression and pathogenesis. Carcinogenesis 30, 894901.

Zhang, C.F., Xia, Y.H., Zheng, Q.F., Li, Z.J., Guo, X.H., Zhou, H.C., Zhang, L.L., Dong, L.P., Han, Y., Liu, Z.E., et al. (2013a). Effect of silencing AEG-1 with small interfering RNA on the proliferation and cell cycle of gastric carcinoma SGC-7901 cells. Zhonghua Zhong Liu Za Zhi 35, 22-27.

Zhang, N., Wang, X., Huo, Q., Li, X., Wang, H., Schneider, P., Hu, G., and Yang, Q. (2013b). The oncogene metadherin modulates the apoptotic pathway based on the tumor necrosis factor superfamily member TRAIL (Tumor Necrosis Factor-related Apoptosis-inducing Ligand) in breast cancer. J. Biol. Chem. 288, 9396-9407. 